\title{
Variabilidad iónica en el complejo fluvial formado por los ríos Pivka, Unica y Ljubljanica (Eslovenia)
}

\author{
Avilés A. ${ }^{1 *}$, M. Novič ${ }^{2}$, V. Clavero ${ }^{\dagger} \&$ F.X. Niell ${ }^{1}$ \\ 1 Departament of Ecology, Faculty of Science, University of Málaga. Campus Universitario de Teatinos \\ S/N. 29071-Málaga, Spain. \\ 2 National Institute of Chemistry. Hajdrihova 19. SI-1001 Ljubljana. Slovenia \\ $\dagger$ In memorian \\ *Corresponding author. e-mail: aviles@uma.es
}

\begin{abstract}
RESUMEN
El Río Ljubanica, situado en su mayor parte en el Dinaric Karst, presenta un curso fluvial sometido a sucesivas subducciones y surgencias. Esta característica, permite estudiar el efecto que el Karst tiene en las variables físico-químicas del agua. La analítica, se ha realizado empleando un cromatógrafo iónico DX500 (DIONEX), capaz de determinar de manera rápida los diferentes aniones y cationes, incluyendo el bicarbonato. Los resultados mostraron importantes incrementos de bicarbonato y magnesio tras penetrar el agua dentro del carst, al tiempo de disminuciones importantes del resto de los iones analizados. Las dos últimas estaciones, tomadas próximas a la desembocadura del río, se presentan con un mayor contenido iónico, reflejando unas características físico-químicas bien diferenciadas de las anteriores.
\end{abstract}

Palabras clave: Carst, cromatografía iónica, río Ljubjianica, Eslovenia

\begin{abstract}
Ljubljanica River, mostly located in the Dinaric Karst, presents a fluvial course subjected to successive subductions and surgences. This characteristic makes possible to study the Karst's effect in the water physico-chemical variables. The analysis was performed with an Ion Chromatograph model DX500 (DIONEX) able to rapidly analyze the different anions and cations, including bicarbonate. The results showed important increases in bicarbonate and magnesium, after water penetrating into the karst, and decreases in the remainder of the analysed ions. The last two stations, located near the river's mouth, presented higher ionic load, that reflecting important physico-chemical differences with the former ones.
\end{abstract}

Keywords: Karst, ion chromatography, Ljubljanica River, Eslovenia

\section{INTRODUCCIÓN}

El agua de los ríos tiene una composición química influenciada por numerosos factores (litológicos, geomorfológicos, climáticos, antropológicos...), condicionando de manera importante la variabilidad espacial de ésta (Hynes, 1975; Margalef, 1983). La meteorización de las rocas de la cuenca es con frecuencia el factor determinante de las características físico-químicas del agua. En pocos lugares, la interacción entre la litología y la composición del agua, es tan evidente como en las regiones cársticas (Milinovi č, 1981; Emmett \& Telfer,
1994). La existencia de numerosas grietas y oquedades por las que circula el agua, puede modificar de forma importante la composición química. La cuenca del río Ljubljanica, es un buen ejemplo de ello. Recoge el agua del extremo NW del Dinaric Karst, siendo un afluente del río Sava, el cual desemboca en el Danubio. El Dinaric Karst es una de las formaciones cársticas más importante de Europa, comprendida entre Italia, Eslovenia y Croacia (Herak \& Stringfield, 1972). Entre las particularidades de esta zona, destacan las numerosas cavidades y la existencia de tres ríos (Pivka, Unica y Ljubljanica), que constituyen en realidad el 


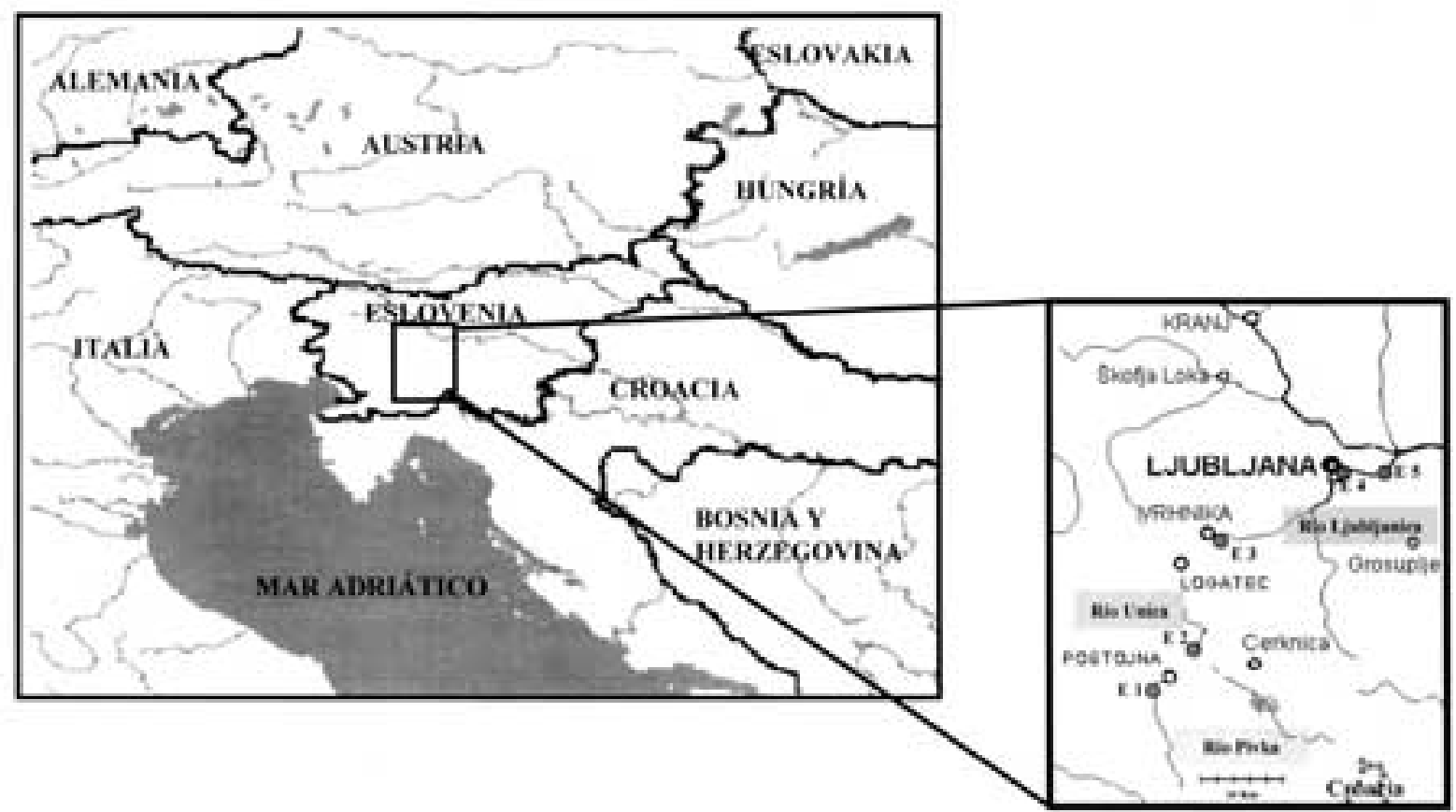

Figura 1. Mapa de la cuenca del río Ljubljanica, incluida las estaciones de muestreo. Map of Ljubljanica Rive's cathment, including in the sampling stations.

curso superficial del mismo complejo fluvial, fluyendo un par de veces bajo la superficie del karst (Bauer et al., 1976). Esto ofrece la oportunidad de estudiar los cambios que sufren las características físico-químicas del sistema fluvial tras los procesos de subducción y el papel "depurador" de esta estructura cárstica.

Una de las técnicas, cada vez más empleada en el estudio de la composición química de los sistemas fluviales, es la cromatografía iónica (Hirayama et al., 1996; Thomas et al., 2002), porque permite una rápida determinación de los principales iones y nutrientes, con lo que los descriptores se caracterizan en poco tiempo con muchos datos. El analizador DX 500, a diferencia del resto de equipos, permite el análisis de bicarbonato, tan necesario para comprender la naturaleza y variabilidad de un complejo fluvial cárstico. El empleo de agua desionizada junto con grupos hidroxilos como fase móvil, en lugar del buffer carbonato / bicarbonato, permite el análisis del bicarbonato con los otros aniones. El uso de este método, constituye una novedad que aporta este estudio.
El objetivo de este estudio es la caracterización química del agua de la cuenca del sistema fluvial Pivka-Unica-Ljubljanica y el efecto que el carst tiene en la composición química del agua superficial en dicho sistema.

\section{MATERIAL Y MÉTODOS}

\section{Área de estudio}

La cuenca del río Ljubljanica se encuentra al SW de Eslovenia, entre el río Sava y la frontera de Eslovenia con Croacia. De sus aproximadamente $1900 \mathrm{~km}^{2}$ de superficie, más del $60 \%$ $\left(1100-1200 \mathrm{~km}^{2}\right)$ se encuentran en el macizo cárstico de Dinaric. Esta formación cárstica, se caracteriza por la existencia de numerosas cuevas, algunas de ellas, con decenas de kilómetros de galerías (e.g. Postojna, Skocjan), numerosos poljes y dolinas, lo que explica que la mayor parte de las aguas sean subterráneas, apareciendo sólo algunos cauces superficiales. La cuenca del Ljubljanica, en verdad es el último tramo de 
un complejo formado por tres cuencas. La primera de ellas la del río Pivka, desaparece en la cueva de Postojna y reaparece a los pocos kilómetros denominándose río Unica. Éste, vuelve a subducir, surgiendo próximo al límite de la región cárstica (Vrhnika) con el nombre de río Lubljanica (Fig. 1). A partir de ese punto, el río fluye superficialmente a través de terrenos más impermeables hasta su desembocadura en el río Sava, tras pasar por el centro de la ciudad de Ljubljana, capital de Eslovenia.

\section{Muestreos y técnicas analíticas}

Para caracterizar el efecto de las sucesivas surgencias, se tomaron cinco estaciones (Fig. 1). La primera de ellas en el río Pivka, cerca de la entrada de la cueva de Postojna. La estación 2, se tomó en el río Unica, a pocos metros de dicha cueva y a $19 \mathrm{Km}$ de la estación 3 (río Ljubljanica). La estación 4 se localizó en el mismo centro de la ciudad de Ljubljana y la última (E 5), próxima a la desembocadura en el río Sava. El estudio se realizó en noviembre de 1999, tras varios días sin nevar y siempre con temperaturas ambientales inferiores a $-1^{\circ} \mathrm{C}$. Las muestras se tomaron por duplicado empleando botes de polietileno (Mackerenth et al., 1978) y se mantuvieron refrigeradas a $4{ }^{\circ} \mathrm{C}$. Todos los análisis se realizaron dentro de las 48 horas siguientes a la recogida.

La analítica de los diferentes iones y cationes se realizó aplicando técnicas de cromatografía iónica, empleando un cromatógrafo modelo DX 500 (DIONEX). Para la analítica de los aniones, se utilizó una columna IonPac AS15 y una columna IonPac CS12A para los cationes, ambas con supresión. Este modelo de cromatógrafo iónico, en particular, incorpora un módulo de generación de eluyente que permite realizar la analítica de aniones empleando agua como fase móvil, enriquecida con grupos hidroxilos liberados en dicho módulo. Para la determinación de los cationes se empleó como solvente ácido sulfúrico $31 \mathrm{mM}$, sin emplear el módulo generador de eluyentes. Las muestras eran previamente filtradas por una malla de $0.45 \mu \mathrm{m}$. El
pH se determinó empleando un sensor modelo CRISSON. Los sólidos en suspensión se analizaron filtrando el agua a través de filtros Whatman de $0.45 \mu \mathrm{m}$. Para las medidas de conductividad, se empleó el detector conductimétrico del mismo cromatógrafo.

Para el análisis de los datos, se realizó un test de correlación de Pearson y un análisis de componentes principales (ACP), empleando los programas informáticos SPSS 10.0 y el MVSP 3.12 , respectívamente.

\section{RESULTADOS}

En la Tabla 1, se presentan los valores medios de las principales variables físico-químicas. Como se observa, la temperatura del agua se incrementa casi siete veces entre las estaciones uno y tres, pasando de 1.2 a $9.4^{\circ} \mathrm{C}$, descendiendo a valores inferiores a $7{ }^{\circ} \mathrm{C}$ en las dos estaciones siguientes. Esto se debe, a que la temperatura ambiental estuvo entre -4 y $-1{ }^{\circ} \mathrm{C}$ durante las horas del muestreo. Tanto los sólidos en suspensión como la conductividad del agua, mostraron descensos importantes al atravesar el carst, aumentando en las dos últimas estaciones. El pH, varió en un rango comprendido entre 7.48 y 7.73 , con un máximo localizado en la estación 2.

El análisis de la composición aniónica y catiónica, refleja una composición dominante de carbonato-cálcico (Fig. 2). Sin embargo, las dos estaciones primeras mostraron una composición mucho más rica en sodio que las tres siguientes, con porcentajes próximos al $40 \%$ frente a valores inferiores al $15 \%$.

Las concentraciones de los diferentes aniones y cationes, presentaron dos tendencias bien diferenciadas (Figs. 3 y 4). La primera, caracterizada por valores bajos en la estación 1 que se incrementan alcanzando el máximo en las dos estaciones últimas (bicarbonato y magnesio) y una segunda, más generalizada, con descensos importantes de concentraciones entre las estaciones 1 y 3 , seguidos de valores más altos en las dos últimas. Destacan el sulfato y el calcio, con valores en las estaciones 4 y 5 comparables, 


\section{Aniones}

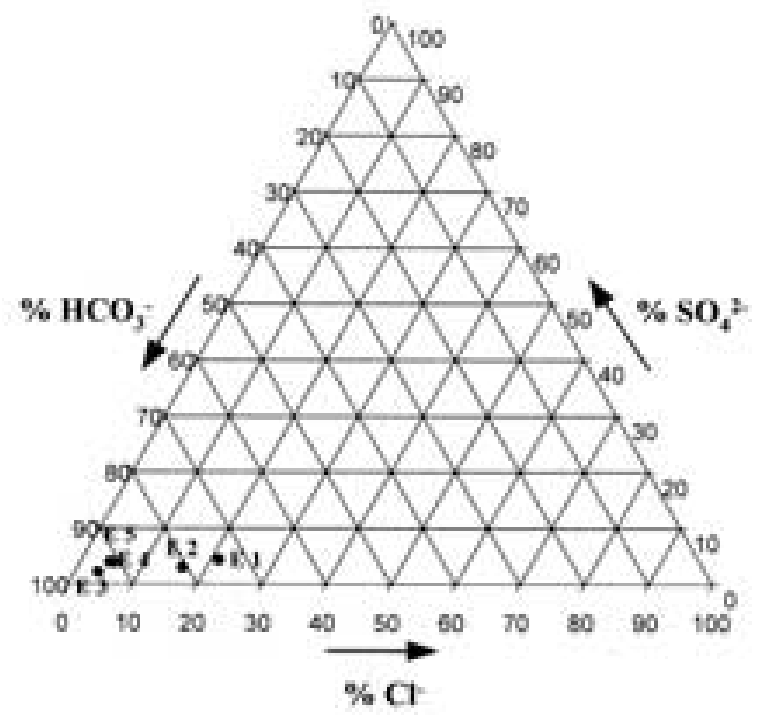

Cationes

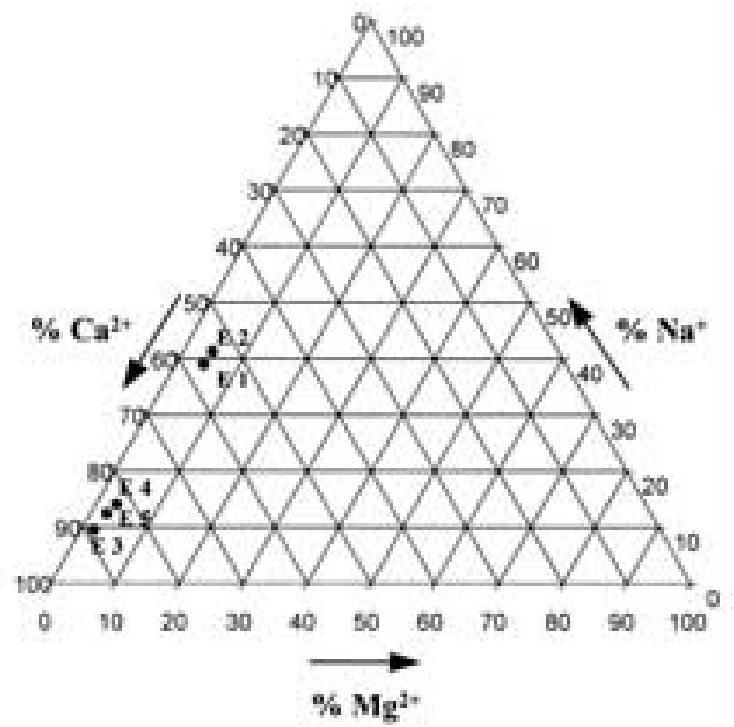

Figura 2. Composición relativa de aniones y cationes (diagrama triangular) del agua en los diferentes puntos de muestreo. Relative anionic and cationic composition (triangular diagram) of the water at the sampling sites.

e incluso superiores, a los registrados en E 1. El empleo de la cromatografía iónica, permite la determinación de los iones fluoruro en bajas concentraciones, fundamental para comprender el ciclo del fosfato en el agua y de su retirada al sedimento en forma de fluorapatito.

En relación con los nutrientes (Fig. 5), el nitrato fue la forma de nitrógeno dominante, con valores que incluso alcanzaron $6.9 \mathrm{ppm}$ en la estación 3. La tendencia seguida por esta variable, muestra un incremento desde la primera estación hasta la ya mencionada, con valores más bajos en las dos últimas. Tendencia opuesta a la obtenida para el nitrito, con un mínimo en $\mathrm{E}$ 3 y máximos en las estaciones finales. El fosfato, se caracterizó por un máximo en E 1, para ir disminuyendo hacia las estaciones aguas abajo.

En general, las correlaciones obtenidas entre las diferentes variables, mostraron bajos valores (Tabla 2). Destacando las correlaciones positivas existentes entre el sodio y cloruro, el magnesio y bicarbonato y entre el sodio y potasio.

\section{Análisis multivariante}

El análisis de componentes principales (ACP) realizado a las cinco estaciones, considerando 16 variables (Tabla 3). El primer eje, explica un $58.6 \%$ de variabilidad, donde se contraponen las variables con un marcado incremento de concentraciones (bicarbonato, magnesio y nitrato) y aquellas caracterizadas por fuertes descensos (sodio, cloruros, fosfato), pudiendo interpretarse este eje como la ordenación de las estaciones de muestreo en el curso del río. El eje 2, que explica un $28.8 \%$, está definido principalmente por el calcio, el sulfato y la conductividad, lo que podría interpretarse como un eje de incremento de carga iónica. Por último, el tercer eje que explica sólo un $9.1 \%$, está definido sobre todo por el $\mathrm{pH}$.

En la figura 6 se presenta la posición de las estaciones y las variables en los dos primeros ejes del ACP. Las dos primeras estaciones se distribuyen en el primer eje con valores negativos, estando las restantes en la zona 
Tabla 1. Valores medios de las principales variables físicoquímicas. Mean values of the main physico-chemical variables.

\begin{tabular}{lccccc}
\hline & E 1 & E 2 & E 3 & E 4 & E 5 \\
& & & & & \\
$\mathrm{T}^{\mathrm{a}}$ del agua $\left({ }^{\circ} \mathrm{C}\right)$ & 1.2 & 6.2 & 9.4 & 6.8 & 6.8 \\
Caudal $\left(\mathrm{m}^{3} \mathrm{~s}^{-1}\right)$ & 3.0 & 8.0 & 16.0 & 21.0 & - \\
$\mathrm{pH}$ & 7.52 & 7.73 & 7.48 & 7.56 & 7.67 \\
$\begin{array}{l}\text { Conductividad } \\
\left(\mu \mathrm{S} \mathrm{cm} \mathrm{cm}^{-1}\right)\end{array}$ & 326.0 & 302.0 & 209.0 & 315.0 & 313.0 \\
$\begin{array}{l}\text { Sólidos en } \\
\text { suspensión }\left(\mathrm{mg} \mathrm{l}^{-1}\right)\end{array}$ & 4.00 & 2.00 & 0.67 & 2.16 & 3.57 \\
\hline
\end{tabular}

positiva. Destaca la posición próxima de las dos últimas estaciones en la zona positiva de ambos ejes, diferenciándose claramente de las tres estaciones previas, con posiciones en la zona negativa del segundo eje.

\section{DISCUSIÓN}

Cada vez son más los estudios realizados en zonas de carst, con el objeto de caracterizar biogeoquímicamente estos sistemas por su fragilidad frente a la contaminación y a su importancia como acuíferos (Kalkhoff, 1995; Winiarski et al., 1995; Kaçaroglu, 1999). Las cuencas cársticas se caracterizan por la existencia de numerosos sumideros y grutas, con sólo unos pocos cursos fluviales en la superficie. Si la consideración de los ríos como "sistemas continuos" (Vannote et al., 1980), está cada vez más cuestionada a favor de una visión mucho más heterogénea (Avilés, 2002), tanto espacial como temporalmente, esta "ruptura de la continuidad", se aprecia de manera clara en los sistemas cársticos. La subducción y posterior surgencia por dos veces del río Ljubljanica, permite observar estos cambios bruscos debido a las interacciones entre el agua y el carst, como si se tratase de un río diferente en cada tramo.

Los trabajos previos existentes en la zona (Varios, 1976; Kogovšek, 1999; Kogovšek et al., 1999), describen una cuenca con numerosos sumideros y complejas redes de drenaje. A pesar de la existencia de otros arroyos y corrientes subterráneas, los cambios observados en las
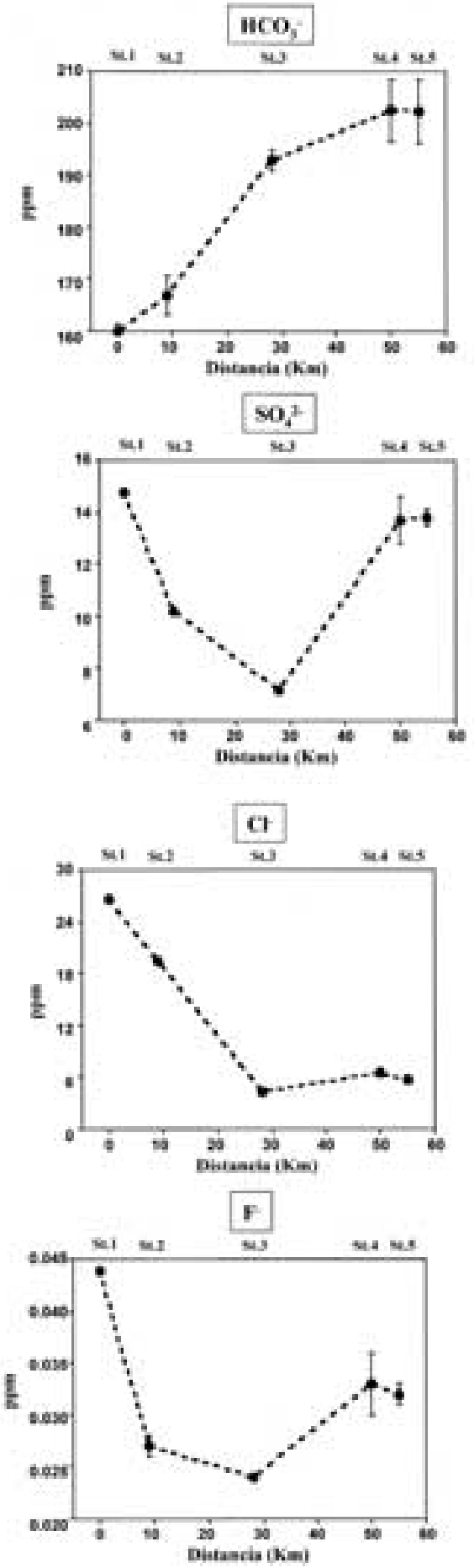

Figura 3. Variación espacial de las concentraciones de los aniones: bicarbonato, cloruro, sulfato a fluoruro, expresadas en $\mathrm{mg} / \mathrm{l}$. Spatial variation of anionic concentrations: hidrogen carbonate, chloride, sulfate and fluoride, expressed as $\mathrm{mg} / \mathrm{l}$. 
Tabla 2. Coeficientes de correlación de Pearson para las diferentes variables analizadas. $\mathrm{n}=5(*$ para $\mathrm{p}=0.05 \mathrm{y} * *$ para $\mathrm{p}=0.01)$. Pearson coefficients correlation for the differrent analized variables. $n=5(*$ for $p=0.05 y * *$ for $p=0.01)$.

\begin{tabular}{|c|c|c|c|c|c|c|c|c|c|c|c|}
\hline & $\mathbf{F}^{-}$ & $\mathrm{Cl}^{-}$ & $\mathrm{NO}_{2}^{-}$ & $\mathrm{HCO}_{3}^{-}$ & $\mathrm{SO}_{4}{ }^{2-}$ & $\mathrm{NO}_{3}^{-}$ & $\mathrm{HPO}_{4}{ }^{2-}$ & $\mathbf{N a}^{+}$ & $\mathbf{K}^{+}$ & $\mathbf{M g}^{2+}$ & $\mathrm{Ca}^{2+}$ \\
\hline $\mathbf{F}^{-}$ & 1 & & & & & & & & & & \\
\hline $\mathrm{Cl}^{-}$ & 0.637 & 1 & & & & & & & & & \\
\hline $\mathrm{NO}_{2}^{-}$ & 0.220 & -0.341 & 1 & & & & & & & & \\
\hline $\mathrm{HCO}_{3}^{-}$ & -0.416 & $-0.955^{*}$ & 0.582 & 1 & & & & & & & \\
\hline $\mathrm{SO}_{4}{ }^{2-}$ & 0.847 & 0.344 & 0.649 & -0.053 & 1 & & & & & & \\
\hline $\mathrm{NO}_{3}^{-}$ & -0.749 & $-0.903 *$ & -0.089 & 0.749 & -0.654 & 1 & & & & & \\
\hline $\mathrm{HPO}_{4}{ }^{2-}$ & 0.771 & $0.881 *$ & -0.389 & -0.833 & 0.345 & -0.728 & 1 & & & & \\
\hline $\mathrm{Na}^{+}$ & 0.724 & $0.992 * *$ & *-0.304 & $-0.924 *$ & 0.419 & $-0.906^{*}$ & $0.922 *$ & 1 & & & \\
\hline $\mathbf{K}^{+}$ & 0.839 & $0.951 *$ & -0.124 & -0.829 & 0.578 & $-0.939 *$ & $0.918^{*}$ & $0.978 * *$ & * 1 & & \\
\hline $\mathbf{M g}^{2+}$ & -0.306 & $-0.914 *$ & 0.640 & $0.992 * *$ & 0.066 & 0.680 & -0.781 & -0.872 & -0.759 & 1 & \\
\hline $\mathrm{Ca}^{2+}$ & 0.309 & -0.037 & 0.510 & 0.233 & 0.669 & -0.235 & -0.201 & -0.005 & 0.068 & 0.321 & 1 \\
\hline
\end{tabular}

diferentes variables, responden al efecto de la circulación subterránea, ya que el Río Pivka es el principal aporte de agua del Río Unica y a su vez éste del Ljubljanica.

Debido a las bajas temperaturas existentes durante el muestreo, fue posible registrar fuertes incrementos al atravesar el agua el carst bajo la superficie. Estos cambios de temperatura unido a los de $\mathrm{pH}$, calcio y carbonato, modifican de manera importante las presiones parciales de $\mathrm{CO}_{2}$, implicadas en las tasas de disolución de los iones de la calcita (Sasowsky \& White, 1993; Herczeg et al., 1997).

La relación entre la composición iónica del agua y la meteorización de las rocas, se ha representado en el diagrama de Gibbs (Fig. 7). Todas las estaciones se distribuyen con rangos de salinidad características de agua dominadas por procesos de meteorización. Si bien, son claras las diferencias existentes entre las dos primeras estaciones y las tres restantes, teniendo las primeras estaciones relaciones $\mathrm{Na}^{+} /\left(\mathrm{Na}^{+}+\mathrm{Ca}^{2+}\right)$ mucho más elevadas. Estas diferencias, junto con los altos valores obtenidos para la mayoría de las variables en la estación primera, se deben a los pequeños vertidos residuales procedentes de las viviendas dispersas existentes en la zona alta del río Pivka, junto con la sal añadida a las carreteras para la fusión de la nieve. Ambos factores determinan, que a pocos kilómetros de E1 se obtuvieran concentraciones de cloruro, sodio y fosfato
Tabla 3. Factores de carga de las variables empleadas en el Análisis de Componentes Principales, para los tres primeros ejes. Loading factors for the first three axes of the Principal Component Analyses.

\begin{tabular}{lrrr}
\hline Variables & Eje I & Eje II & Eje III \\
\hline Distancia & 0.251 & 0.270 & 0.095 \\
Temperatura del agua & 0.302 & -0.116 & -0.129 \\
pH & -0.012 & 0.164 & -0.747 \\
Conductividad & -0.184 & 0.349 & -0.127 \\
Sólidos en suspensión & -0.202 & 0.315 & 0.038 \\
$\mathrm{~F}^{-}$ & -0.246 & 0.204 & 0.349 \\
$\mathrm{Cl}^{-}$ & -0.311 & -0.057 & -0.118 \\
$\mathrm{NO}_{2}{ }^{-}$ & 0.075 & 0.384 & 0.095 \\
$\mathrm{HCO}_{3}{ }^{-}$ & 0.282 & 0.180 & 0.176 \\
$\mathrm{SO}_{4}^{2-}$ & -0.155 & 0.383 & 0.162 \\
$\mathrm{NO}_{3}{ }^{-}$ & 0.293 & -0.121 & 0.134 \\
$\mathrm{HPO}_{4}{ }^{2-}$ & -0.293 & -0.089 & 0.254 \\
$\mathrm{Na}^{+}$ & -0.316 & -0.032 & -0.033 \\
$\mathrm{~K}^{+}$ & -0.314 & 0.040 & 0.063 \\
$\mathrm{Mg}^{2+}$ & 0.263 & 0.224 & 0.206 \\
$\mathrm{Ca}^{2+}$ & -0.013 & 0.389 & -0.257 \\
\hline$\% \mathrm{Varianza}^{-}$ & 58.6 & 28.8 & 9.1 \\
\hline
\end{tabular}

superiores a 145, 112 y $24 \mathrm{mg} / 1$, respectívamente. Estas altos valores, pueden explicar el comportamiento opuesto existente entre este sistema y otros parecidos como el existente en el Macizo Central Francés. Mientras que en el ejemplo francés, el carst produce incrementos importantes de calcio y tenues de sodio y cloruros (Vaute et al., 

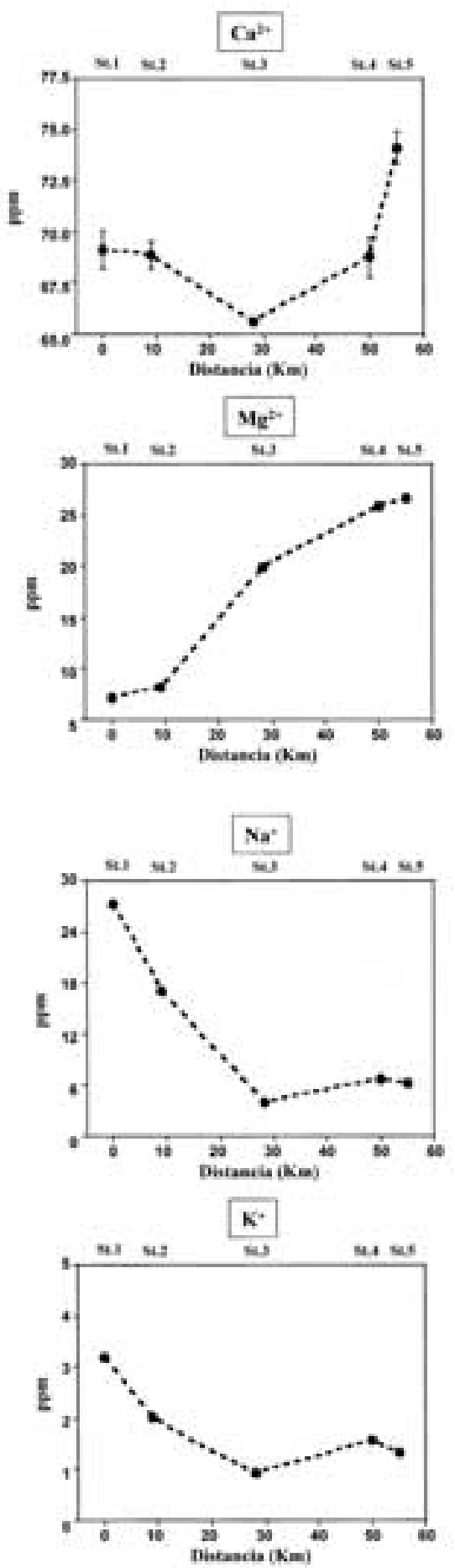

Figura 4. Variación espacial de las concentraciones de los cationes: calcio, sodio, magnesio y potasio, expresadas en $\mathrm{mg} / \mathrm{l}$. Spatial variation of cationic concentrations: calcium, sodium, magnesium and potasium, expressed as $\mathrm{mg} / \mathrm{l}$.
1997), en nuestro caso, se produce un descenso importante de las concentraciones de estos iones y de casi todos los analizados.

A pesar de ser este un sistema cárstico antiguo y de ser sistemas caracterizados por su escasa interacción con el agua que fluye subsuperficialmente (Varios, 1976), los resultados muestran un importante efecto como acumuladores de iones, retirando importantes concentraciones del agua. Sólo el carbonato y el magnesio, incrementaron de manera notable sus concentraciones, debido al efecto de disolución de la calcita.

Las estaciones últimas localizadas próximas al río Sava, mostraron incrementos importantes de bicarbonato, sulfato, calcio y magnesio con respecto a las concentraciones obtenidas en $\mathrm{E} 3$. Aunque entre E 3 y E 5, el río discurra siempre por la superficie, es clara la interacción con la litología y el aporte de otros afluentes ricos en estas sales. Sin embargo, el efecto de la ciudad de Ljubljana, incluida la depuradora de aguas residuales existente entre las estaciones 4 y 5 , no produce impactos patentes. Los descensos de concentraciones obtenidos entre las estaciones 3 y 5 para el nitrato y fosfato, así lo muestran. Este escaso efecto antropológico se relaciona con el elevado caudal del río, frente al volumen vertido por la depuradora. El efecto de la actividad biológica como causa de la reducción de nutrientes, se descarta debido a las bajas temperaturas existentes en el agua $\left(<7{ }^{\circ} \mathrm{C}\right)$, lo que reduce de manera importante esta actividad. La ausencia de las concentraciones de amonio, debido a la metodología empleada, afecta notablemente al estudio de los nutrientes, al ser esta la forma de nitrógeno de más rápida asimilación por los organismos (e.g. Stanley \& Hobbie, 1981), además de ser la forma más abundante en las aguas residuales.

Los resultados del ACP, ponen de manifiesto también las diferencias existentes entre las tres primeras estaciones y las dos siguientes (Fig. 6). Las primeras, se disponen marcando una tendencia clara con la distancia y disminuyendo su carga iónica (valores cada vez más negativos del segundo eje). Mientras que las dos últimas, presentan mayores cargas iónicas. 

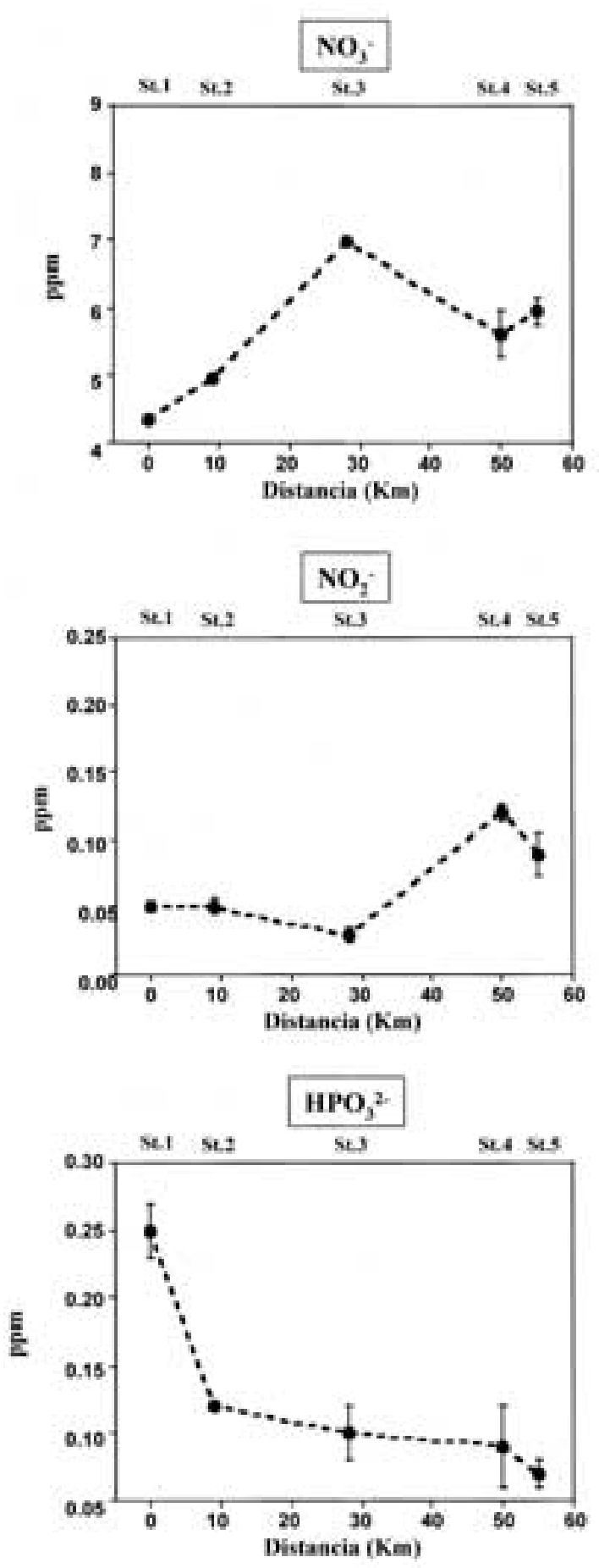

Figura 5. Variación espacial de las concentraciones de los nutrientes: nitrato, nitrito y fosfato, expresadas en $\mathrm{mg} / \mathrm{l}$. Spatial variation of the nutrients concentrations: nitrate, nitrite and phosphate, expressed as $\mathrm{mg} / \mathrm{l}$.

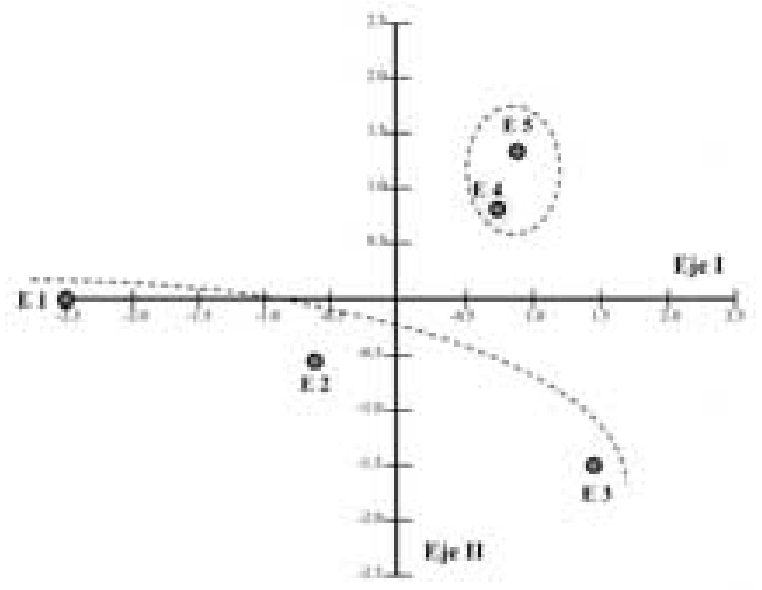

Figura 6. Representación de las estaciones en los dos primeros ejes del ACP. Stations' representations on the first two components of PCA.

\section{CONCLUSIONES}

La cuenca del río Ljubljanica, se presenta como un sistema complejo, dominado por las interacciones entre el agua y las rocas que componen el carst. Los procesos de precipitación y solubilización que se producen en las aguas subterráneas, modifican las concentraciones registradas de casi todas las variables analizadas. Las altas concentraciones iónicas existentes en la zona alta del río Pivka, condicionan de manera importante el papel depurador de este sistema cárstico, frente a lo obtenido en otros sistemas parecidos.

El empleo del cromatógrafo iónico DX 500, ha permitido el análisis de manera rápida de los principales iones, incluyendo el bicarbonato. Sin embargo, la cromatografía tiene el inconveniente de no poder determinar las concentraciones de amonio, debido al solapamiento con el pico de sodio, cuando los valores de éste son altos. Esta limitación, puede tener una gran importancia, sobre todo en las dos últimas estaciones, al ser el amonio la principal forma de nitrógeno procedente de las aguas residuales urbanas, lo que nos permitiría diferencias con más claridad los efectos naturales de los antropogénicos. 


\section{AGRADECIMIENTOS}

Este trabajo se ha realizado gracias a la beca concedida por la Junta de Andalucía para la estancia realizada en el Instituto Nacional de Química de Ljubljana (Eslovenia), desde octubre a diciembre de 1999, estando este trabajo en el marco del proyecto de investigación titulado "Cambios en los ciclos biogeoquímicos y en la fisiografía de una cuenca de pequeño tamaño", CICYT AMB 99-1088. Agradecemos la atención y ayuda prestada por todo el personal perteneciente al laboratorio de analítica de dicho instituto en el aprendizaje de las técnicas de cromatografía iónica.

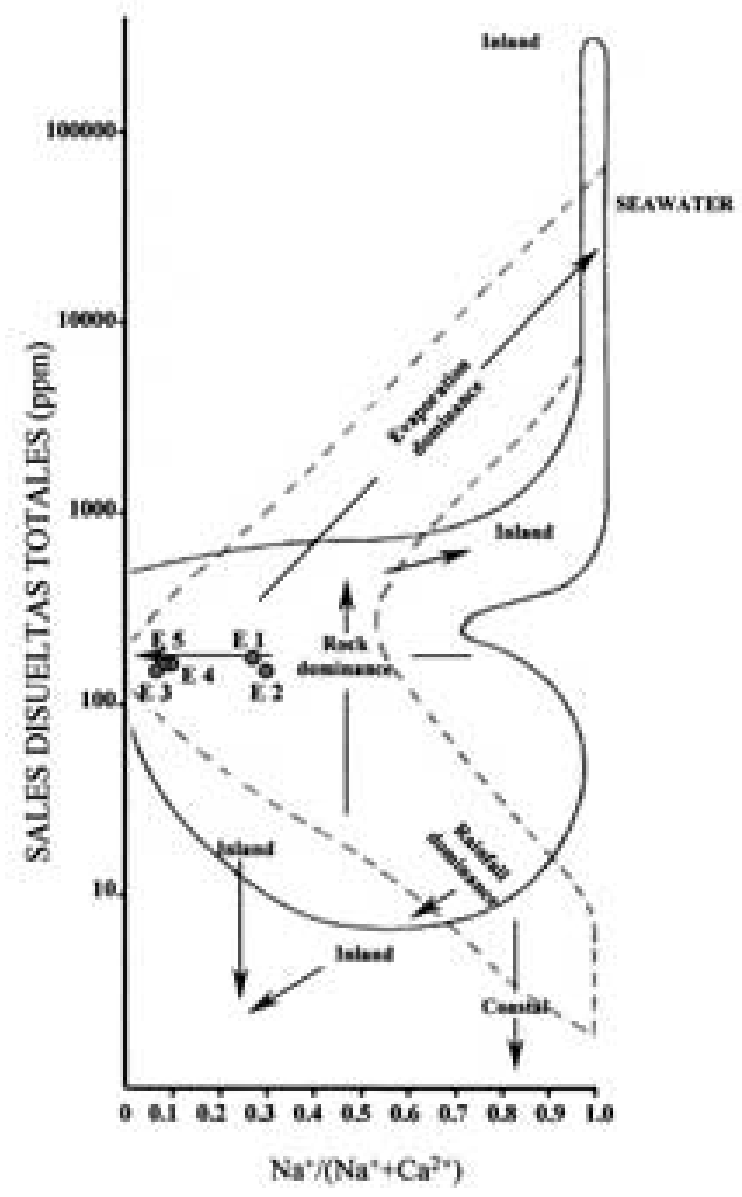

Figura 7. Proyección de las estaciones en el diagrama de Gibbs modificado (Gibbs, 1992). Sampling scores on the modified Gibbs diagram (Gibbs, 1992).
Este artículo es un homenaje a la memoria de Vicente Clavero, que asistió como invitado a la reunión de Barcelona sobre Eutrofización de lagos y embalses en países Iberoamericanos y cuyo fallecimiento no le permitió completar el manuscrito que estaba preparando sobre las tasas de retirada de fósforo en el embalse de Charco Redondo.

\section{BIBLIOGRAFÍA}

AVILÉS, A. 2002. El papel del sector fluvial en la biogeoquímica del Río Palmones (Los Barrios, Cádiz). Tesis doctoral. Universidad de Málaga. $191 \mathrm{pp}$.

BAUER, F., H. BEHRENS, F. BERNOT, B. BUKVI, S. BAUER, M. DECHANT, F. DROBNE, R. GOSPODARI, P. HABI, H. HÖTZL, F. HRIBAR, W. KÄSS, M. ROLBEZEN, P. KRIVIC, F. LEWICKI, V. MAURIN, J. MISEREZ, H. MOSER, M. MOZETI, A. PIINIM, M. PIRŠ, V. RAJNER, D. RANK, W. STICHLER, M. TOPLIŠEK, M. TRATNIK, R. UDOV, R. VERBOSEK, J. G. ZÖTL, M. ZUPAN y K. IBRIK. 1976. Underground water tracing (investigations in Slovenia 1972-1975). R. Gospodarič \& P. Hablič (eds.). Institute Karst Research. 312 pp

EMMETT, A. J. \& A. L. Telfer. 1994. Influence of karst hydrology on water quality management in southeast South Australia. Envirom. Geol., 23: 149-155.

Areply to the comment of Eilers et al.

HERAK, M. \& T. V. STRINGFIELD. 1972. Karst. Important karst regions of Northern Hemisphere. Elsevier Publ. Comp. N.Y.

HERCZEG, A. L., F. W. J. LEANEY, M. F. STADTER, G. L. ALLAN \& L. K. FIFIELD. 1997. Chemical and isotopic indicators of point-source recharge to a karst aquifer, South Australia. $J$. Hydrol., 192: 271-299.

HIRAYAMA, N., M. MARUO, H. OBATA, A. SHIOTA, T. ENYA \& T. KUWAMOTO. 1996. Measurement of fluoride ion in the river-water flowing into Lake Biwa. Wat. Res., 30(4): 865-868.

HYNES, H. B. N. 1975. The stream and its valley. Verh. Internat. Verein. Limnol., 19:1-15.

KAÇAROGLU, F. 1999. Review of groundwater pollution and protection in karst areas. Wat. Air Soil Poll., 113: 337-356. 
KALKHOFF, S. J. 1995. Relation between streamwater quality and geohydrology during baseflow conditions, Roberts Creek watersheed. Clayton County, Iowa. Wat. Resour. Bull., 31(4): 593-604.

KOGOVŠEK, J. 1999. New knowledge about the underground water dranaige in the northern part of Javorniki mountains (High Karst). Acta Carsologica, 28(1): 161-200.

KOGOVŠEK, J., M. KNEZ, A. MIHEVČ, M. PETRIC, T. SLABE \& S. ŠEBELA. 1999. Env. Geol., 38(1):69-76.

MACKERETH, F. J. H., J. HERON \& J. F. TALLING.1978. Water analysis. Some methods for limnologists. Freshwater Biological Association. Scientific Publication $n^{\circ} 36$. Cumbria, L.A.

MARGALEF, R. 1983. Limnología. Omega. Barcelona.

MILINOVIČ, P. 1981. Karst Hydrogeology. Wat. Res. Publ., Littleton, Colorado, USA.

RASOWSKY, I. D. \& W. B. WHITE. 1993. Geochemistry of the Obey River Basin, north-cen- tral Tennessee: a case of acid mine water in a karst drainage system. J. Hydrol., 146: 29-48.

STANLEY, D. W. \& J. E. HOBBIE. 1981. Nitrogen recycling in a North Carolina coastal river. Limnol. Oceanogr., 26: 30-42.

THOMAS, D. H., M. REY \& P. E. JACKSON. 2002. Determination of inorganic cations and ammonium in environmental waters by ion chromatography with a high-capacity cation-exchange column. J. Chromatog., 956(1/2): 181-186.

VANNOTTE, R. L., G. W. MINSHALL, K. W. CUMMINS, J. R. SEDELL \& C. E. CUSHING. 1980. The river continium concept. Can. J. Fish. Aquat. Sci., 37: 130-137.

VAUTE, L., C. DROGUE, L. GARRELLY \& M. GHELFENSTEIN. 1997. Relations between the structure of storage and the transport of chemical compounds in karstic aquifers. J. Hydrol., 199: 221-238.

WINIARSKI, T., O. THOMAS \& C. CHARRIER. 1995. Analysis of the sapatial and temporal variations in the water quality of karstic aquifer using UV spectrophotometry. J. Cont. Hydrol., 19: 307-320. 